\title{
DOI: https://doi.org/10.46296/gt.v4i8edesp.0038
}

\section{FACTORES PROTECTORES Y DE RIESGO EN FAMILIAS EN SITUACIÓN DE VULNERACIÓN DE DERECHOS}

\section{PROTECTIVE AND RISK FACTORS IN FAMILIES IN SITUATIONS OF RIGHTS VIOLATION}

\author{
Valdivieso-Cobeña Martin Augusto ${ }^{\text {; }}$ Escobar-Delgado Gustavo Rafael ${ }^{2}$ \\ ${ }^{1}$ Maestría Académica con Trayectoria de Investigación en Psicología, Mención Psicoterapia. \\ Instituto de Posgrado de la Universidad Técnica de Manabí, UTM. Portoviejo, Ecuador. Correo: \\ martinvaldivieso69@hotmail.com. ORCID ID: https://orcid.org/0000-0003-1635-7656 \\ ${ }^{2}$ Universidad Técnica de Manabí, UTM. Portoviejo, Ecuador. Correo: \\ gustavo.escobar@utm.edu.ec. ORCID ID: https://orcid.org/0000-0002-0732-7757
}

\begin{abstract}
Resumen
Los factores protectores y de riesgo en familias en situación de vulneración de derechos es una herramienta en la que funcionalmente convergen el criterio de los profesionales y el de las familias para identificar fortalezas y debilidades familiares con el fin de realizar un diagnóstico de la situación familiar y comunitaria. Esta investigación tiene como objetivo prevenir, preservar, fortalecer o restablecer los vínculos familiares para evitar la separación innecesaria del niño, niña o adolescente de su entorno familiar y comunitario. La metodología implementada fue de naturaleza cuantitativa, estudio no experimental de temporalidad transversal. Se analizaron los factores generales, competencias parentales y seguridad familiar; datos recogidos a través de la aplicación de la ficha de ponderación de factores protectores y de riesgo basada en North Carolina Family Assesment Scale encontrando claras fortalezas, una importante presencia de problemas serios y moderados en los factores específicos valorados. Así, esta investigación resulta estratégica para la ulterior implementación de planes de intervención psicoterapéuticos que brinden apoyo a las familias, en la intención de abordar los problemas serios y moderados encontrados y arquimédicamente sugerir patrones de crianza y educación integral que eviten la separación innecesaria de los niños, niñas y adolescentes de su entorno familiar y comunitario.
\end{abstract}

Palabras clave: Competencias parentales; seguridad familiar; factores protectores y de riesgo.

\begin{abstract}
Protective and risk factors in families in a situation of rights violation is a tool in which the criteria of professionals are combined with that of families to identify strengths and weaknesses of families in order to diagnose the situation. family and community. This research aims to prevent, preserve, strengthen or reestablish family ties to avoid unnecessary separation of the child or adolescent from their family and community environment, the methodology implemented was quantitative in nature, Non-experimental study of cross-sectional temporality in which the general factors parental competencies and family safety were analyzed, which were collected through the application of the weighting sheet of protective and risk factors based on the North Carolina Family Assessment Scale, finding clear strengths, an important presence of serious and moderate problems in the specific factors assessed. Through this research, it was possible to obtain a general result for the implementation of family psychotherapeutic intervention plans to address the serious and moderate problems found in this research, obtaining a favorable upbringing that avoids the unnecessary separation of children and adolescents from their environment, an important presence of serious and moderate problems in the specific factors assessed. Through this research, it was possible to obtain a general result for the implementation
\end{abstract}

Información del manuscrito:

Fecha de recepción: 13 de julio de 2021.

Fecha de aceptación: 27 de septiembre de 2021.

Fecha de publicación: 12 de noviembre de 2021. 
of family psychotherapeutic intervention plans to address the serious and moderate problems found in this research, obtaining a favorable upbringing that avoids the unnecessary separation of children and adolescents from their environment, an important presence of serious and moderate problems in the specific factors assessed. Through this research, it was possible to obtain a general result for the implementation of family psychotherapeutic intervention plans to address the serious and moderate problems found in this research, obtaining a favorable upbringing that avoids the unnecessary separation of children and adolescents from their environment.

Keywords: Parental competencies; family safety; protective and risk factors.

\section{Introducción}

Actualmente, las medidas de protección en la infancia giran en torno a la separación del niño, niña o adolescente de su entorno natural de crecimiento proporcionando ambientes de desarrollo óptimos, tales como entidades de acogimiento o al cuidado de progenitores/ cuidadores. En varias ocasiones se ha evidenciado que algunas de esas medidas carecen de alternativas preventivas; es decir, no se interviene para evitar que los niños, niñas 0 adolescentes sean separados de su familia biológica, sino más bien para que estos abandonen el núcleo familiar, indicando que las medidas adoptadas priorizan la protección de los niños, niñas o adolescentes y no la familia que son el origen central de sus dificultades sociales; por lo cual, los problemas siguen persistiendo y se reflejan en sus entornos, tales como centros educativos, sociales y sobre todo en sus relaciones interpersonales.

El estudio factores protectores y de riesgo en familias en situación de vulneración de derechos se enmarcan en el proyecto apoyo familiar de la Fundación Fadinnaf que tiene como objetivo prevenir, preservar, fortalecer o restablecer los vínculos familiares para evitar la separación innecesaria del niño, niña o adolescente del núcleo familiar que se encuentran en situación de riesgo de vulneración de derechos, identificado como negligencia que no sea causado por razones económicas, maltrato psicológico o físico, violencia intrafamiliar que no constituya delito y en el caso de una situación de discapacidad leve o moderada de sus padres que por su condición no pueden ejercer el 
cuidado y protección necesaria para

el bienestar del niño, niña 0 adolescente. A su vez busca restablecer sus vínculos y garantizar el derecho a la convivencia familiar y comunitaria como parte de la aplicación y seguimiento de la política pública de niñez y adolescencia (Ministerio de Inclusión Económica y Social, 2019).

Ergo, la investigación responde a la necesidad de identificar los factores protectores y de riesgo en las familias que se encuentran en situación de vulneración de derechos, dicho riesgo puede conllevar a una posible institucionalización del niño, niña o adolescente a través de una medida judicial de acogimiento institucional como recurso en el caso de que no exista familia biológica o ampliada hasta el tercer grado de consanguinidad que pueda asumir su cuidado y protección.

\section{Materiales y métodos}

La aplicación de esta medida para los niños, niñas y adolescentes no es la más adecuada, según las investigaciones, algunas entidades de acogimiento institucional carecen de recursos materiales $y$ profesionales, además, sus servicios se basan en un enfoque asistencialista. Estas razones ubican a los niños, niñas y adolescentes en una situación de vulnerabilidad que afecta su desarrollo integral (Loja \& Quituisaca, 2017). Así, la recurrencia de esta precaria situación no alienta el espíritu institucional para la atención efectiva de la integridad de las personas.

La afectación a su desarrollo integral implica un conjunto de secuelas a nivel psicológico, físico y cognitivo. Esto sugiere que la institucionalización de niños, niñas y adolescentes maltratados, respecto de su no institucionalización, tendría un efecto negativo aún mayor sobre el desarrollo cognitivo y socioemocional (Deambrosio et al., 2017).

La preexistencia de estos efectos negativos en este vulnerable colectivo, potencian la importancia del proyecto de apoyo familiar cuya finalidad cardinal es evitar la separación innecesaria del niño, niña o adolescentes del núcleo familiar por medio de la determinación de los factores protectores y de riesgo de la familia, favoreciendo la identificación de fortalezas y debilidades que son 
abordadas en el proceso de intervención psicoterapéutico familiar para restituir derechos, bienestar y felicidad.

Esto representa un aporte significativo para la comunidad científica que está trabajando ardua y sistemáticamente en la prevención de la institucionalización innecesaria y desinstitucionalización de niños, niñas y adolescentes debido a que de esta manera se puede establecer un punto de partida para las futuras investigaciones e intervenciones en este ámbito.

El instrumento de ponderación de factores protectores y de riesgo evalúa los factores: entorno con factores socio-económicos, culturales y comunitarios en que viven las familias, competencias parentales como las capacidades prácticas de la madre -padre, para cuidar, proteger y educar a sus hijas/os, las interacciones familiares la forma de como la familia se relaciona, seguridad familiar ausencia o presencia de riesgos para la seguridad y protección del niño/a o adolescente dentro de la familia, ambivalencia padre-cuidador versus hijos que se genera debido a una inconsistencia en las habilidades emocionales de sus cuidadores, los progenitores no les ofrecen una confianza en cuanto a la consistencia de sus respuestas, los niños suelen mostrarse ansiosos, impredecibles, la red de apoyo o recursos del sistema familiar que pueden ponerse en movimiento para hacer frente a diversas situaciones de riesgo que se presentan en los hogares y la preparación para la culminación del proceso de interacción son factores importantes para decidir el cierre (North Carolina Family Assessment Scale for Reunification, 2007).

El análisis de las publicaciones científicas sobre el tema de los factores protectores y de riesgo, considera los estudios de los efectos negativos que produce la institucionalización en los niños, niñas y adolescentes (Loja \& Quituisaca, 2017) y (Deambrosio et al., 2017) realizan un aporte respecto a estos efectos, debido a que estos son el motivo de la creación del proyecto apoyo familiar, el que al ser relativamente nuevo en el contexto latinoamericano y ecuatoriano posee una limitada cantidad de investigaciones que evalúen el desempeño y el impacto que tiene el 
proyecto apoyo familiar en las familias que se encuentran en riesgo de vulneración de derechos, constituye un grave problema a escala mundial dada su elevada prevalencia, así como a los serios daños a corto y largo plazo que genera en el desarrollo infantil (Contreras Taibo et al., 2018).

Perú cuenta con un programa de formación y apoyo familiar que está basado en una metodología participativa y experiencial similar al proyecto aplicado en Ecuador en consecuencia, es importante tener en cuenta la evaluación de la implementación del programa de formación y apoyo familiar con familias peruanas (Maya \& Hidalgo, 2016).

Independientemente del país de aplicación el proyecto apoyo familiar tiene como finalidad que el niño se mantenga en su núcleo familiar y la intervención por medio de la identificación de los factores protectores y factores de riesgo para fortalecer las habilidades de cuidado, la comunicación y la resiliencia en los miembros de la familia.

Se pretende conseguir que los Estados sean conscientes de la necesidad de proporcionar a los padres y las madres apoyos psicoeducativos suficientes para cumplir sus importantes responsabilidades en la crianza y educación de sus hijos/as, especialmente en aquellos que viven en circunstancias de riesgo psicosocial (Martín et al., 2013).

Desde la nueva concepción de los niños como sujetos de derecho y no como objeto de protección se busca la problemática del cuidado y visibilizar a los niños/as y adolescentes como actores sociales que participan de la producción de discursos sociales sobre las relaciones familiares (Chávez Ibarra \& Vergara del Solar, 2018).

En la primera etapa de la investigación se identificaron a las familias con niños, niñas y adolescentes en situación de riesgo de vulneración de derechos, identificado como negligencia que tengan medidas administrativas emitidas por la Junta cantonal de protección de derechos del cantón Portoviejo.

Durante la segunda etapa se aplicó la ficha factores protectores y de riesgo para conocer detalles de la 
situación de las familias desde la filosofía construccionista social, no existiendo verdades absolutas y considerando que cada persona observará de diferentes formas a las familias y sus situaciones. En la detección de fortalezas y debilidades, el diálogo familiar es muy importante, como también lo es el criterio de los profesionales, quienes, con voz asertiva, sin radicalizar verdades, comparten y destacan la trascendencia de las fortalezas y de los retos familiares.

La tercera etapa consistió en el análisis de la información de las áreas evaluadas por la ficha factores protectores y de riesgo, mediante el programa estadístico Statistical Package for the Social Sciences (SPSS), como también en realizar comparaciones entre los factores competencias parentales y seguridad familiar.

Desde la teoría del apego, es decir, desde el vínculo afectivo que le entrega al niño una sensación de seguridad, confianza y placer, se puede llegar al concepto de competencias parentales y así entender la relevancia que éste tiene en la relación paterno filial. El apego está directamente relacionado con la capacidad que tengan los padres de satisfacer, proteger y cuidar a sus hijos, por ende, está directamente relacionado con la capacidad que tengan los padres de ser competentes (Conde, A. C., \& Gotschlich, E. S., 2015).

La ficha de factores protectores y de riesgo, toma en cuenta los siguientes factores específicos: capacidad de los padres para cuidar y proteger a los hijos, supervisión de los niños, niñas - adolescentes, prácticas disciplinarias adecuadas, recursos de la familia que promueven el desarrollo del niño/a - adolescente, salud metal de los progenitores o cuidadores, salud física de los progenitores o cuidadores, el uso de drogas o alcohol de los progenitores o cuidadores, mientras que el factor seguridad familiar representa la ausencia o presencia de riesgos para la seguridad o protección del niño/a o adolescente dentro de la familia, los factores específicos valorados son: presunción de abuso físico, sexual, emocional en el niño/a - adolescente, presunción de negligencia en el niño/a adolescente, presunción de violencia doméstica, salud mental, comportamiento y desempeño 
escolar del niño/a - adolescente, relación con el progenitor o cuidador, con los hermanos y con sus pares y la cooperación y motivación a permanecer en la familia.

Se realizó una investigación de tipo no experimental de temporalidad transversal. Como método empírico se aplicó la ficha de ponderación de factores protectores y de riesgo basada en la "North Carolina Family Assessment Scale for Reunification" (NCFAS-R) que es una escala tipo Likert.

Se tomó como población a las 17 familias del proyecto apoyo y custodia familiar de la Fundación Fadinnaf y como muestra a las 10 familias del proceso apoyo familiar en el año 2020-2021, según reporte remitido por el equipo técnico de la Fundación Fadinnaf de la ciudad de Portoviejo conformado por psicóloga clínica, trabajadora social y facilitadora familiar. Como criterio de inclusión se consideró a las familias con medidas administrativas emitidas por la Junta cantonal de protección de derechos del cantón Portoviejo, familias nucleares 0 monoparentales con hijos biológicos y familias con salud mental suficiente para comprender y responder a los requerimientos de este estudio; mientras que, como criterio de exclusión a las familias que no dieron su carta de consentimiento para la participación en el estudio; y, como criterios de eliminación a las familias que cambiaron su lugar de residencia que no sea la ciudad de Portoviejo.

Los criterios éticos emanan de la carta de consentimiento de la investigación al representante legal de la Fundación Fadinnaf, carta de consentimiento de la familia participante, la confidencialidad de la información mediante la aplicación de códigos a las familias participantes en el software estadístico SPSS de manejo de los autores, además de declaración de no haber conflictos de intereses.

\section{Resultados}

En el gráfico de las competencias parentales de la Ficha de factores protectores y de riesgo North Carolina Family Assessment Scale instrumento aplicado a 10 familias. 
Tabla 1. Competencias parentales.

\begin{tabular}{cccccc}
\hline & Frecuencia & Porcentaje & $\begin{array}{c}\text { Porcentaje } \\
\text { válido }\end{array}$ & $\begin{array}{c}\text { Porcentaje } \\
\text { acumulado }\end{array}$ \\
\hline Válido & Problema Serio (PS)" & 2 & 20,0 & 20,0 & 20,0 \\
\cline { 2 - 6 } & Problema Moderado (PM) & 1 & 10,0 & 10,0 & 30,0 \\
\cline { 2 - 6 } & Problema Leve (PL) & 1 & 10,0 & 10,0 & 40,0 \\
\cline { 2 - 6 } & Leve Fortaleza (LF) & 2 & 20,0 & 20,0 & 60,0 \\
\cline { 2 - 6 } & Clara Fortaleza (CF) & 4 & 40,0 & 40,0 & 100,0 \\
\hline Total & 10 & 100,0 & 100,0 & \\
\hline
\end{tabular}

De acuerdo a la tabla competencia parentales de las 10 familias a las que se aplicó la ficha de factores protectores y de riesgo, el $40 \%$ se encuentra en clara fortaleza lo que significa resultados satisfactorios correspondientes a la evaluación de las capacidades de los progenitores para cuidar, proteger y educar a los hijos; mientras que, el $20 \%$ presenta leve fortaleza en los factores evaluados lo que revela que tienen buena capacidad para sobrellevar situaciones adversas; el $10 \%$ se posiciona en problema leve, lo que representa surgimiento de conflictos; sin embargo, la familia presenta recursos positivos para resolverlos; otro $10 \%$ de las familias presenta problemas moderados lo que evidencia que la familia intenta resolver los problemas y sabe cómo, pero existe alguna dificultad $u$ oposición que impide la resolución de los mismos; no obstante busca ayuda.

El $20 \%$ de las familias se sitúa en problema serio; esto denota que la familia no encuentra solución a los conflictos y es renuente a buscar ayuda, por lo que presenta poca capacidad para cuidar y proteger a los hijos, una pobre supervisión de los niños, niñas - adolescentes, prácticas disciplinarias inadecuadas, ausencia de recursos de la familia que promueven el desarrollo del niño/a - adolescente, afectación en la salud mental y física y uso de drogas o alcohol de los progenitores.

Tabla 2. Seguridad Familiar

\begin{tabular}{|c|c|c|c|c|c|}
\hline & & Frecuencia & Porcentaje & $\begin{array}{c}\text { Porcentaje } \\
\text { válido }\end{array}$ & $\begin{array}{l}\text { Porcentaje } \\
\text { acumulado }\end{array}$ \\
\hline \multirow[t]{3}{*}{ Válido } & Problema Moderado (PM) & 2 & 20,0 & 20,0 & 20,0 \\
\hline & Clara Fortaleza (CF) & 8 & 80,0 & 80,0 & 100,0 \\
\hline & Total & 10 & 100,0 & 100,0 & \\
\hline
\end{tabular}


Los resultados de la tabla seguridad familiar indican que el $80 \%$ de las familias presentan recursos que posibilitan la ausencia de abuso físico, sexual y emocional, así como también la negligencia y violencia doméstica lo que influye positivamente en la salud mental, comportamiento, desempeño escolar del niño, niña o adolescente. Se colige que las relaciones con el progenitor y con los hermanos, pares (amigos, clubes deportes) han incidido significativamente para que haya la cooperación, motivación, confianza y seguridad, evitando la separación innecesaria de la familia biológica y asegurando la funcional permanencia en la familia de origen.

El $20 \%$ de las familias se ubica en problema moderado, es decir, tienen ciertas dificultades para mantener la seguridad familiar, exponiendo a los/as hijos/as a situaciones de vulneración de derechos debido a que la resolución de problemas en la familia se torna difícil o presenta oposición por parte de alguno de los miembros; sin embargo, están interesados en la ayuda externa que les permita la superación de los problemas que tienen que ver con actitudes negligentes hacia los niños, niñas 0 adolescentes y violencia domestica entre los progenitores 0 cuidadores. Las causas de este fenómeno, de acuerdo con lo expresado en la ficha de factores protectores y de riesgo, son la falta de tiempo destinado al cuidado de los hijos, la alta demanda de tareas domésticas por carga familiar, desconocimiento a los derechos de la niñez y adolescencia y tipología de maltrato infantil generando un diagnóstico desfavorable.

\section{Discusión}

A partir de los resultados expuestos, se corrobora asociación de los factores evaluados de competencia parentales y seguridad familiar existiendo una correlación significativa. Dentro de los factores específicos de competencias parentales se pondera características funcionales de las figuras parentales encontrando una clara fortaleza en salud mental de los progenitores lo que potencia su habilidad parental, y/o resolución exitosa de problemas; por otro lado, se detecta una leve fortaleza en supervisión de los niños, niñas o adolescentes lo cual demuestra que 
existe una frágil supervisión para la edad, como por ejemplo fijar límites para las actividades de los niños, niñas, adolescentes, una línea base para las apropiadas prácticas disciplinarias y guía bajo la tutela de los progenitores; también, se identifican problemas leves en la capacidad de los padres para cuidar y proteger a los hijos, quienes requieren de la práctica permanente y reforzamiento de la comunicación, medidas de prevención y exposición hacia las experiencias traumáticas; además, existen problemas moderados en recursos de la familia lo que quiere decir que existe falta de apoyo o sobre involucramiento de los progenitores en oportunidades para los niños, niñas y adolescentes, tales como deportes, lecciones musicales y/o salidas a terreno, evidenciándose que los progenitores no estimulan ni desincentivan el involucramiento de los hijos e hijas en estas actividades.

Por otro lado, en los factores específicos del factor general de seguridad familiar se identifica una clara fortaleza en ausencia de abuso físico, sexual y emocional, en el niño, niña o adolescente, relación con el progenitor o cuidador, hermanos y pares (amigos, clubes, deportes, etc.), esto expresa que en las familias nunca han ocurrido incidentes, quejas, pruebas de abuso físico, sexual y emocional, o han existido pero la familia ha tenido recursos de afrontamiento. En cuanto a la relación con el progenitor o cuidador, hermanos y pares, se evidencia que existe acercamiento entre ellos. Existen problemas serios en ausencia/ presencia de negligencia del niño, niña o adolescente que significa que hay incidentes, quejas, pruebas de negligencia infantil por cuidadores que no han sido notificadas 0 abordadas, o se han resuelto insatisfactoriamente; asimismo, problemas serios ausencia de violencia doméstica entre los progenitores que se refiere a incidentes, quejas, arrestos por violencia doméstica.

Se presentan problemas moderados y serios en un porcentaje importante de los factores de competencias parentales, lo que se evidencia en la dinámica relacional en la familia, se destacó presencia de importantes conflictos entre las figuras parentales en la dinámica cotidiana del hogar. Estos conflictos parecen irradiar hacia un abanico de procesos del 
sistema familiar que pueden impactar negativamente en los niños: recreación e interacciones lúdicas escasas, relaciones con redes de apoyo precarias y débiles, conflictos recurrentes con otras personas externas a la familia, estos resultados, influyen en la seguridad familiar.

Claramente se logra apreciar que existen ciertas similitudes y diferencias en algunos de los aspectos específicos de los factores generales de competencias parentales y seguridad familiar; por ejemplo, en el rol parental se identifica que no existen problemas en su salud mental, sin embargo, en seguridad familiar en cuanto a los niños, niñas o adolescentes se evidencia algunas problemáticas en su salud mental; por otro lado, se detecta que las figuras de progenitores o cuidadores suelen poner reglas en cuanto a prácticas disciplinarias, pero, en ocasiones se exceden los límites pudiendo desencadenarse casos de violencia doméstica.

(Deza, 2015) en la investigación sobre factores de riesgos y protección en niños, niñas y adolescentes en situaciones de pobreza de instituciones educativas de Villa el Salvador, señala que es posible actuar oportunamente en cuando se identifican las condiciones de factores de riesgo, pues si bien no son determinantes se pueden predecir en situaciones críticas para los niños, niñas y adolescentes, especialmente el maltrato, descuido, desarraigo afectivo hasta la ausencia física y emocional de los progenitores.

\section{Conclusiones}

- La figura parental desempeña un rol vital en el desarrollo integral de la personalidad; es incuestionable su incidencia en la esfera emocional de niños, niñas y adolescentes, teniendo como escenario medular la familia.

- $\quad$ El presente estudio permitió identificar una serie de temáticas importantes en el entorno de las familias nucleares. En primer lugar, encontramos el factor general de competencias parentales de los padres hacia sus hijos evidenciando a su vez cada uno de los factores específicos que este se clasifica y notando que existe clara fortaleza en salud mental de los progenitores o cuidadores, leve fortaleza en 
supervisión de los niños, niñas o adolescentes; hay una línea base en prácticas disciplinarias adecuadas, problemas leves en capacidad de los padres para cuidar y proteger a los hijos; así como también en supervisión de los niños, niñas o adolescentes $\quad \mathrm{y}$ disciplinarias y problemas moderados en recursos de la familia que promueven el desarrollo.

- $\quad$ El factor general de seguridad familiar revela que existe clara fortaleza en aspectos como ausencia de abuso físico, sexual y emocional, en el niño, niña o adolescente, relación con el progenitor, hermanos y pares (amigos, clubes, deportes, etc.); también se identificó problemas serios en los factores específicos de presencia de negligencia, violencia doméstica, salud mental, comportamiento y desempeño del niño, niña, adolescente y en la cooperación y motivación a permanecer en la familia.

- En concordancia con el objetivo de este estudio que fue el de prevenir, preservar, fortalecer 0 restablecer los vínculos familiares para evitar la separación innecesaria del niño de su núcleo familiar, es oportuno el diseño de planes de trabajo y modelos de intervención como apoyo familiar para el fortalecimiento de las competencias parentales que aportarán a la seguridad familiar como prevención al abandono o institucionalización de los niños, niñas y adolescentes, garantizando que vivan en un entorno familiar y comunitario, restituyendo de esta manera sus derechos y garantizando el bienestar y la felicidad.

- $\quad$ Queda abierta la posibilidad de que está investigación sea un punto de partida para futuras indagaciones en el área de protección especial y desinstitucionalización de los niños, niñas y adolescentes para evitar los efectos negativos que produce la experimentación de vivencias adversas tempranas debido a que causa vulneración de derechos y problemas en el desarrollo integral de la niñez, para así evitar afectaciones en su vida y lograr desarrollar adultos resilientes y afectivamente responsables. 


\section{Bibliografía}

Chávez Ibarra, P. B., \& Vergara del Solar, A. C. (2018). El problema del cuidado desde la perspectiva de los niños(as): Análisis crítico del discurso sobre el cuidado entre padres e hijos(as). Universitas Psychologica, 17(3), 1-11. https://doi.org/10.11144/Javer iana.upsy17-3.pcpn

Conde, A. C., \& Gotschlich, E. S. (2015). Reflexiones en torno a las competencias parentales: Una propuesta de definición operacional. 35-51. https://doi.org/10.7770/RCHD YCP-V6N1-ART887

Contreras Taibo, L., Paulsen Gutierrez, C., \& Gómez Muzzio, E. (2018). Graves vulneraciones de derechos en la infancia y adolescencia: Variables de funcionamiento familiar.

Universitas Psychologica, 17(3), 1-13. https://doi.org/10.11144/Javer iana.upsy17-3.gvdi

Deambrosio, M., Gutiérrez de Vázquez, M., Universidad Adventista del Plata, Argentina., Arán-Filippetti, V., Consejo Nacional de Investigaciones Científicas y Técnicas (Conicet), Argentina., Román, F., \& Universidad de la Costa, Colombia y Fundación Hippocampus, Argentina. (2017). Efectos del Maltrato en la Neurocognición. Un Estudio en Niños Maltratados Institucionalizados y no Institucionalizados. Revista Latinoamericana de Ciencias Sociales, Niñez y Juventud, 16(1), 239-253. https://doi.org/10.11600/1692 $715 \times .16114$

Deza, S. (2015). Factores de riesgo y protección en niños y adolescentes en situación de pobreza de instituciones educativas en Villa El Salvador. Av.psicol. vol 23, 231-240

Loja, W. F. S., \& Quituisaca, A. A. S. (2017). Modalidades alternativas de acogimiento de niños, niñas y adolescentes en el contexto ecuatoriano. 16, 17.

Martín, J. C., Cabrera, E., León, J., \& Rodrigo, M. J. (2013). La Escala de Competencia y Resiliencia Parental para madres y padres en contextos de riesgo psicosocial. Anales de Psicología, 29(3), 886-896. https://doi.org/10.6018/anales ps.29.3.150981

Maya, J., \& Hidalgo, M. V. (2016). Evaluación de la implementación del Programa de Formación y Apoyo Familiar con familias peruanas. 34, 10.

Ministerio de Inclusión Económica y Social. (2019). Norma Técnica de Protección Especial. 
https://www.inclusion.gob.ec/

wp-

content/uploads/2019/01/nor

ma_tecnica_modalidades_alt

ernativas0314.pdf

North Carolina Family Assessment

Scale for Reunification.

(2007). Escala y definiciones. 